\title{
Comparison of Multi-class and Fusion of single-class SegNet model for Classifying Karst Wetland Vegetation using UAV Images
}

\author{
Bolin $\mathrm{Fu}^{1}$, Man Liu ${ }^{1}$, Hongchang $\mathrm{He}^{1 *}$, Donglin Fan ${ }^{1 *}$, Lilong Li ${ }^{1}$, Liangke Huang ${ }^{1}$, Ertao Gao ${ }^{1}$ \\ 1 College of Geomatics and Geoinformation, Guilin University of Technology, No.12 Jiangan \\ Street, Guilin 541004, China \\ * Correspondence: dlfan@glut.edu.cn, hhe@glut.edu.cn ; Tel.: 17307735350, 13626246111.
}

\begin{abstract}
Karst wetlands are being seriously damaged, and protecting it has become an important matter. Karst vegetation is the essential component of wetland and plays an important role in in the ecological functions of wetland ecosystems. Classifying karst vegetation is important for karst wetlands protection and management. This paper addressed to classify karst vegetation in Huixian National Wetland Park, located in China using the improved SegNet Deep-Learning Algorithm and UAV images. This study proposed a method to fuse single-class SegNet models using the maximum probability algorithm for karst vegetation classification, and compared with object-based RF classification and multi-class SegNet classification, respectively. This paper evaluated the performance of multi-class SegNet model and fusion of single-class SegNet model with different EPOCH values for mapping karst vegetation. A new optimized post-classification algorithm was proposed to eliminate the stitching traces caused by SegNet model prediction. The specific conclusions of this paper include the followings:(1) fusion of four single-class SegNet models produced better classification for karst wetland vegetation than multi-class SegNet model, and achieved the highest overall classification accuracy $(87.34 \%)$; (2) The optimized post-classification algorithm was able to improve prediction accuracy of SegNet model, and it could eliminate splicing traces; (3) The karst wetland vegetation classifications produced by single-class SegNet model outperformed multi-class SegNet model, and improved classification accuracy(F1-Score) between 10\% 25\%;(4)The EPCOH values and textural feature important impact on karst wetland vegetation classifications. The SegNet model with EPCOH 15 achieved greater classification accuracy(F1-Score) than the model with EPOCH 5 or 10. The textural feature improved improves the capability of the SegNet model for mapping karst vegetation;(5) Fusion of single-class SegNet models and objectbased RF model could provide high classifications results for karst wetland vegetation, and both achieved greater $87 \%$ overall accuracy.
\end{abstract}

Keywords: karst wetland mapping, SegNet model, UAV images, fusion model, texture feature

\section{Introduction}

Wetlands are some of the most productive habitat and ecosystem types on the planet [1-2]. Wetlands are not only natural water filters and homes of species but also a nature-based tourism destination [3-4]. Due to the importance of wetlands, World Wide Fund for Nature (WWF), governments and other organizations are committed to protecting wetlands through the Ramsar Convention. Wetlands include karst wetlands, coastal wetlands, mangroves, estuary wetlands, etc. Among them, karst wetlands (taking the Huixian karst wetlands in Guangxi province, China as an example) have important environmental adjustment roles and ecological benefits. For example, it can maintain water sources, purify water quality, store floods and droughts, and maintain biodiversity. At the same time, their wetland features are rare and are of great research value wetland type [5-7]. Because of the rapid development of society and the economy and the impact of human activities, karst wetlands have been seriously damaged. Its area is shrinking and biodiversity has been decreasing. Vegetation is an important part of a wetland ecosystem. The distribution of wetland 
vegetation is the basis for protecting, managing and restoring wetlands. Therefore, to prevent the loss of wetlands and protect existing wetlands, it is necessary to count and monitor their land uses and the spatial distribution of wetland vegetation. The current research on karst wetlands mainly analyzes and monitors the organic matter or microorganism content in the soil to analyze the distribution and pollution of karst wetland[8-11]. Gil-Márquez et al.[9] studied the chemical and isotopic evolution of groundwater in an evaporite karst plateau (including wetland areas and saline to hyper-saline springs) located at southern Spain. Chamberlin et al.[10] constructed mass balances of both calcium and phosphorus for two watersheds in Big Cypress National Preserve in southwest Florida (USA) to evaluate the time scales over which its striking landscape pattern developed. Several studies have analyzed the distribution and change of karst vegetation using field survey and field sampling [12-13]. However, in situ floristic mapping and data collection usually are expensive, labor intensive and even dangerous due to difficulties of navigating wetlands.

Remote sensing technology has been used to classify vegetation in marsh wetlands, estuary wetlands, coastal wetlands and other wetlands [14-20]. Lane et al. [17] explored and evaluated the utility of a newly launched high-resolution, eight-band satellite system (Worldview-2; WV2) for identifying and classifying freshwater deltaic wetland vegetation and aquatic habitats in the Selenga River Delta of Lake Baikal. Julie et al. [18] classified wetland vegetation formations at a 1:10,000 scale using eight dual-polarization TerraSAR-X images (HH/VV) was acquired in 2013 during dry and wet seasons. Franklin et al. [20] used combinations of Radarsat-2 synthetic aperture radar (SAR) quadpolarization data and Landsat-8 Operational Land Imager (OLI) spectral response patterns to be distinguished circumboreal Canadian bogs and fens by differences in soil, hydrology, vegetation, and morphological features. These studies have confirmed that remote sensing provides a practical means in data collection and wetland mapping to inform management. Unmanned aerial vehicles (UAVs) offer a cost-effective, flexible approach with the ability to provide the finer spatial and temporal resolution needed to adequately discriminating and mapping salt marsh species[21] and mangrove species [22-23]. However, there has been relatively little efforts to classify karst vegetation using UAV imageries.

Shallow machine learning algorithms, such as maximum likelihood, k-nearest neighbor (KNN), support vector machine (SVM), decision tree and random forest (RF) algorithms have long been the main methods for discriminating wetland vegetation[24-29]. However, these algorithms spend a lot of time and effort to tune model hyper-parameters and select optimal input features. Transfer learning produced by shallow machine learning algorithms also has a large learning cost. Recently, deep learning has provided an effective method to automatically learn and identify features from large amounts of data[30], which is the main difference compared to the traditional shallow machine learning algorithms. With the development of deep learning theory, many deep learning architectures, such as convolutional neural network (CNN), fully convolutional network (FCN), SegNet, PSPNet and DeepLabV3 plus networks have achieved state-of-the-art performance in land use/land cover classification[31], rivers extraction[32] and buildings detection[33]. Yang[32] designed a new $\mathrm{CNN}$ architecture that can extract useful high-level features of water bodies from input data in the context of urban background, and achieved the average overall accuracy is $99.14 \%$. These studies confirmed that a powerful CNN requires a large amount of training data [34-35]. At present, the important semantic labeling dataset of training data for deep-learning model has produced based on manual visual interpretation, which usually consumes a lot of time and labor intensive. The accuracy of semantic labeling is greatly affected by the expert knowledge. Hence there is a need for development of a method of producing semantic labeling that can be undertaken repeatedly and inexpensively. When identified the single-class object, most deep learning architectures can produce over $95 \%$ classification accuracy. However, there is still a lack of (1) research on fusion of single-class deep-learning model for multi-class image classifications, and (2) comparison of multi-class deeplearning model and fusion of single-class deep-learning models for mapping karst wetland vegetation. The objective of this study was to fill the gaps by classification of karst wetland vegetation using SegNet deep-learning algorithm and UAV imageries.

The main objectives of this study are: (1) A new approach by combining object-based RF classification model with visual interpretation produced semantic labeling dataset. (2) Attempted to 
construct a high-precision deep-learning model for classifying karst wetland vegetation using SegNet network. (3) proposed an optimized post-classification algorithm to eliminate the splicing traces caused by model prediction of SegNet network. (4) explored the effects of training parameters $\mathrm{EPOCH}$ values and texture feature on karst wetland vegetation classification. (5) Evaluated the perform of multi-class SegNet model and fusion of single-class SegNet models on karst wetland vegetation mapping in Huixian National Wetland Park.

\section{Study Area and Data Source}

\subsection{Study Area}

Huixian National Wetland Park $\left(25^{\circ} 01^{\prime} 30^{\prime \prime} \mathrm{N} \sim 25^{\circ} 11^{\prime} 15^{\prime \prime} \mathrm{N}, 110^{\circ} 08^{\prime} 15^{\prime \prime} \mathrm{E} \sim 110^{\circ} 18^{\prime} 00^{\prime \prime} \mathrm{E}\right)$ is located in Guilin city, Guangxi province, southeast China. The total length of wetland park is about 6 kilometers from east to west. The total width is about 2.8 kilometers from north to south. It is the most evocative and largest karst wetland in China and even the world's low-lying and low-altitude karst areas. This wetland feature and its surroundings are extremely rare in the global peak forest karst plain features and are typically wetlands of significant research value [36-37]. Since the 1950s, under the impact of human activities and the lack of effective management and protection measures, original karst wetlands have been continuously undermined. The water body of wetland has gradually been shrinking. Extensive wetland areas of were reclaimed as paddy field and fish ponds in the past 50 years, which have resulted in the decrease of biodiversity. At the same time, invasive species such as water hyacinth have seriously threatened the few remaining wild plant species in the wetlands.

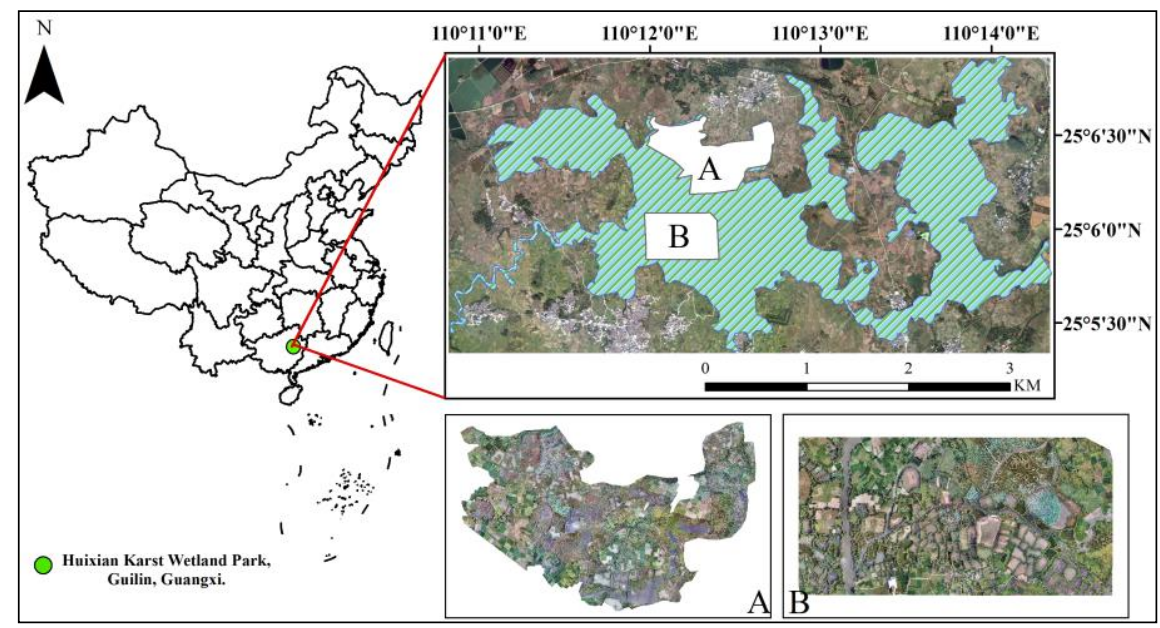

Figure 1 the study area and the location ( Area A and B are UAV ortho-images)

Through field investigation and analysis of the basic characteristics of Huixian National Wetland Park, the core areas A and B of Huixian National Wetland Park were selected as the test area(Figure 1), which covered the original and complete karst wetland vegetation types, such as lotus flower, water hyacinth, bamboo and linden. The ecological appearance is less damaged by human activities. Karst wetland vegetation usually was surrounded by rivers and lakes.

\subsection{UAV image Collections and Preprocessing}

The original UAV aerial images were collected using a DJI Phantom 4 Pro professional V2.0 with a 1-inch 20MP CMOS sensor. The lens angle is set to $-90^{\circ}$ nadir, the front-overlap is $80 \%$, and the side overlap is $75 \%$. The collection time was at noon from 10:00 am to 3:00 pm (UTC/GMT +8.00) during 10-20 July, 2018. The weather was clear and cloudless. The drone flew at an altitude of $90 \mathrm{~m}$ above ground level with the $3.4 \mathrm{~cm}$ ground sampling distance(GSD). Prior to UAV flight, the latitude and 
longitude coordinates of ground place markers(GCPs) were obtained using a Trimble R6 GPS-RTK with positioning accuracy $\pm 2 \mathrm{~cm}$ (Trimble, $\mathrm{CA}$, USA) for georeferencing and photo alignment during UAV images processing.

The collected aerial images were processed by Pix4D Mapper software, ArcGIS software and ENVI software to obtain the digital ortho-images(DOM) of the study area. The Pix4D Mapper software perform the following operations: (1) Importing of original aerial image, POS data including latitude and longitude coordinates and flight attitude, and GCPs. (2) Checking the images quality, remove images with the end-overlap rate of less than $80 \%$ and the side-overlap rate of less than $70 \%$ to ensure the accuracy of aerial triangulation and densification network. (3) automatic matching of image data pairs, aerial triangulation, and block adjustment to generate dense point cloud data in the research area. (4) Using dense point cloud data to build a TIN triangle network in the research area to obtain three-dimensional surface model in the research area. (5) the DOM images of study area were obtained by using the three-dimensional surface model and aerial triangulation parameters, and through the image coloring, stitching and clipping processing. This study used the tool Seamless Mosaic tool in ENVI 5.4 software to complete the mosaic and histogram matching of DOM image.

Filed measurements were carried out from 10-25 July, 2018in the study area. Each sample plot was taken with a multi-angle photography and recorded geographic coordinates using using the Trimble R6 GPS-RTK. Finally. The karst vegetation types in this paper were classified four categories including karst water, karst grass, karst broad-leaved forest, and karst aquatic flora. The sample data was randomly divided into two parts: $2 / 3$ as a training data and $1 / 3$ as a test data using the RStudio software (Table 1). The training data was utilized to produce object-based RF classification for karst wetland vegetation. The testing data were used to verify the classification accuracy of object-based RF and SegNet models.

Table 1 the samples of training datasets data and testing sample sets

\begin{tabular}{ccccccc|c}
\hline \multirow{2}{*}{ Region } & Types & $\begin{array}{c}\text { Karst } \\
\text { water }\end{array}$ & $\begin{array}{c}\text { Karst } \\
\text { grass }\end{array}$ & $\begin{array}{c}\text { Karst broad- } \\
\text { leaved forest }\end{array}$ & $\begin{array}{c}\text { Karst aquatic } \\
\text { flora }\end{array}$ & $\begin{array}{c}\text { Other } \\
\text { types }\end{array}$ & Total \\
\hline \multirow{2}{*}{ Area A } & Training Data & 34 & 58 & 32 & 52 & 22 & 198 \\
& Testing Data & 20 & 20 & 20 & 20 & 20 & 100 \\
\multirow{2}{*}{ Area B } & Training Data & 42 & 48 & 36 & 60 & 28 & 214 \\
& Testing Data & 20 & 20 & 20 & 20 & 20 & 100 \\
\hline
\end{tabular}

\section{Methods}

To construct a high-precision SegNet model for identifying karst wetland vegetation, this study attempted to build multi-class SegNet model and fusion of single-class SegNet model with different EPOCH values and texture features. Therefore, the flowchart of this study mainly included three parts (Figure 2): (1) UAV images collection and preprocessing. (2) combination of object-based RF classifications and visual interpretation was used to produce semantic labeling dataset.(3) construction of multi-class SegNet model and fusion of four single-class SegNet models with different EPOCH values (5, 10 and 15) and texture features. (4) development of post-classification algorithm and conditional random field (CRF) for optimizing SegNet-based classification results. 


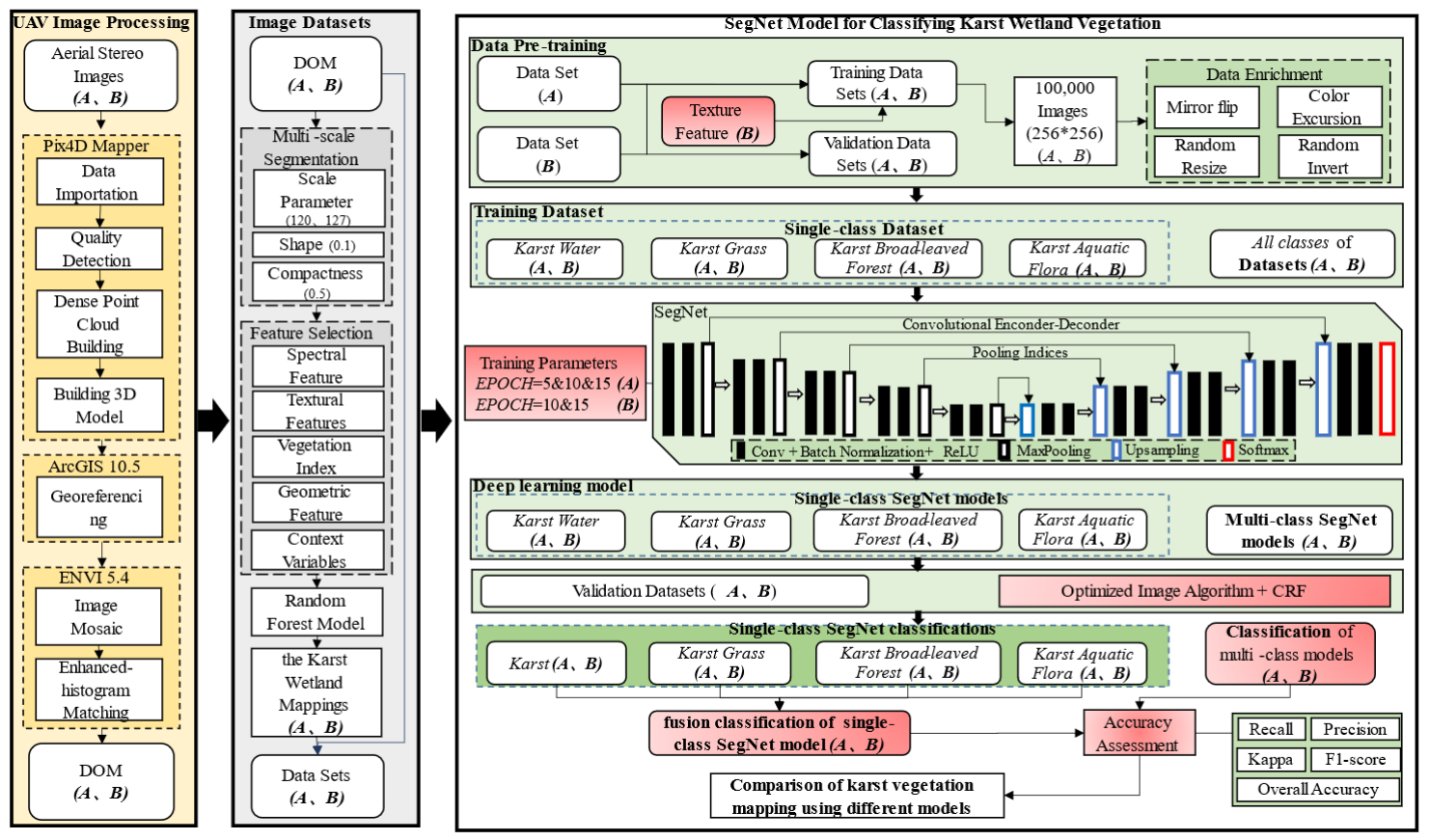

Figure 2 Flowchart of classifying karst vegetation based on SegNet models

\subsection{Combination of Object-based RF classification model and Visual Interpretation Produced semantic labeling dataset}

RF is a machine learning algorithm proposed by Breiman Leo and Adele Cutler in 2001, which combines Breiman's "bagging" idea and random selection of features[38]. The RF algorithm has the advantages of high classification accuracy, strong generalization ability, and can provide the importance estimation of variables. It has been widely used in wetland vegetation identification [3940]. In this study, the object-based RF algorithm was firstly used to classify karst wetland vegetation. The overall accuracy (OA)and kappa coefficient of RF model for Area A and B was both over 0.90 calculated using the test data. The vegetation categories wrongly classified by RF model were corrected by visual interpretation with the field measurements. The corrected classification results of the study area were as semantic labeling dataset(Figure 3(b) and 3(d)) for SegNet-based classifications.

There are three main steps to implement the object-based RF classification for karst wetland vegetation: image segmentation, feature selection, and creation of RF classification model. The specific process was as follows:

(1) Image segmentation. This paper used multi-scale segmentation algorithm to segment the UAV images. Three segmentation parameters, i.e., color/shape weights, smoothness/compactness weights and a scale parameter were considered. An automated segmentation scale parameter selection tool (ESP2) based on secondary development of eCognition Developer software was used to determine the optimal scale parameters of Area $\mathrm{A}(120)$ and Area $\mathrm{B}(127)$ by 500 iterative segmentations.

Then the trial and error method was also used to conduct a multi-scale segmentation experiment for finding the optimal combination of color/shape weight and smoothness/compactness weight. We tried different combinations for segmenting UAV image, such as 0.1/0.9, 0.3/0.7, 0.5/0.5, and 0.7/0.3. Finally, the experiments determined that the optimal combination was 0.1/0.9 and 0.5/0.5.

(2) Feature selection, this study comprehensively considers five types of image features, including spectral feature, texture feature, shape features, and context variables. All image features were calculated using eCognition 9.4 software. The grey-level co-occurrence matrix (GLCM) was used to calculated the textural features, including mean, variance, homogeneity, contrast, dissimilarity, entropy, etc. The Recursive feature elimination(RFE) algorithm from the Boruta package in RStudio was used to select optimal input features of Area A(49) and B (56) for RF classification. 
(3) RF classification model. The training parameters of object-based RF classification included the max number of trees(ntree), the number of split variables(mtry). In this study, 1200 classification trees were constructed. The values for the other parameters were default values 8 for the best split variables determined by the square root of the total number of features. The model training and classification of $\mathrm{RF}$ algorithm for karst wetland vegetation were implemented using the randomForest package.

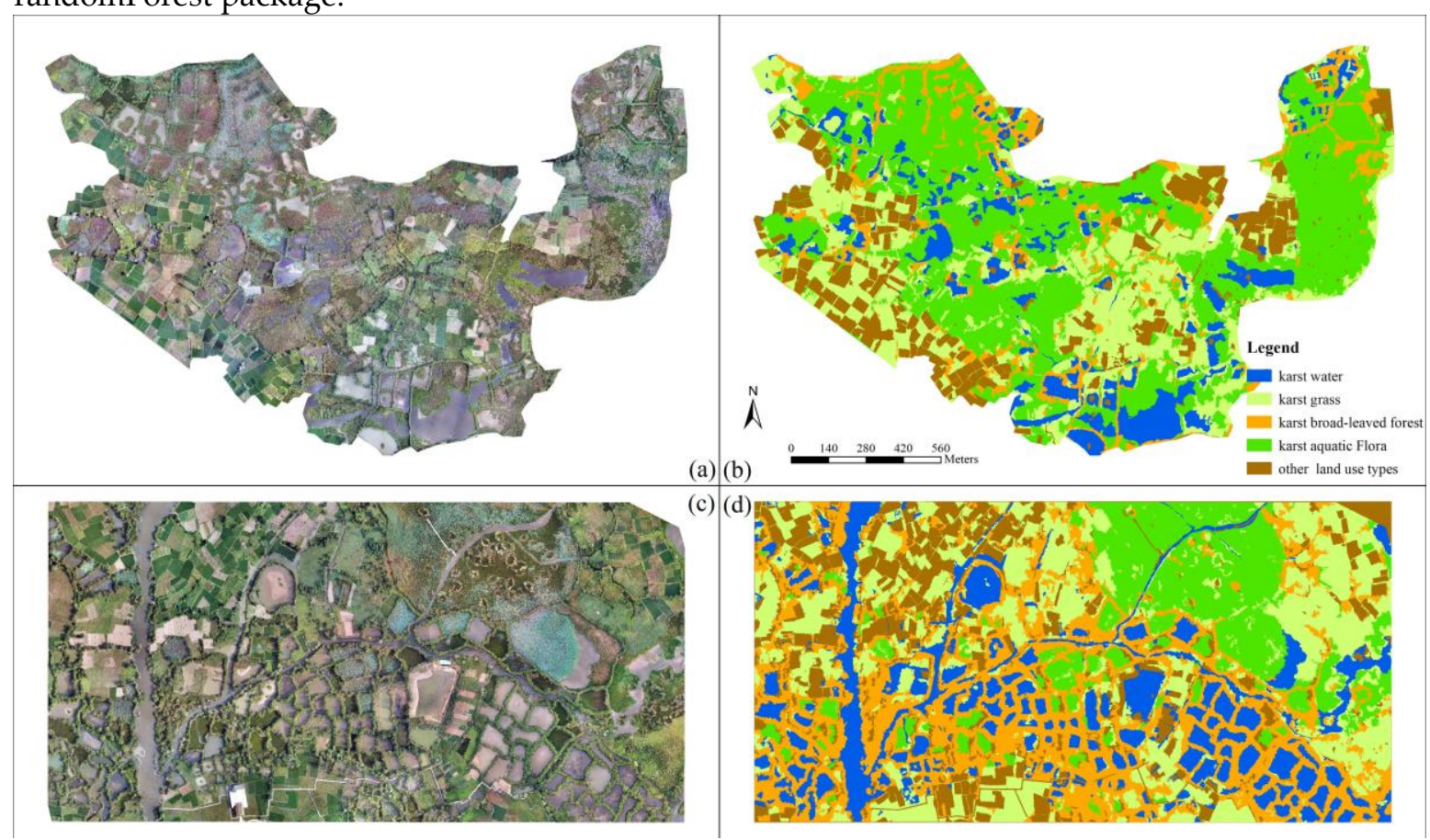

Figure 3 The semantic labeling dataset of karst wetland for SegNet classification (a) DOM image of area A. (b) semantic labeling dataset of Area A, 10550*5320 pixels. (c) DOM image of area B. (d) semantic labeling dataset of area B, $11290 * 7176$ pixels.)

\subsection{SegNet-based Classification Model of Karst Wetland}

SegNet architecture is designed to be an efficient architecture for pixel-wise semantic segmentation, which mainly composed of encoder network, decoder network, and pixel-wise classification layer [41]. The layers are followed by batch normalization [42] and the rectified linear unit(ReLU)activation function. It provides a good balance between accuracy and computational cost. Moreover, symmetrical architecture and combination of the pooling/upsampling of SegNet architecture are very effective for precise re-localization of features[43-46], which is intuitively crucial for remote sensing image classification.

Textural information can help improve the spectral separability of wetland vegetation[47]. A reported study found that as the number of iterations increases, the loss function of Deeplab V3 plus decreased rapidly and gradually stabilized, and the classification accuracy of model is rapidly improved and stabilized[48]. Therefore, in order to construct a high-precision SegNet model for extracting karst wetland vegetation, this paper added the textural features into the input image features, and developed four single-class SegNet models, two multi-class SegNet models and two fusion of single-class SegNet models with different $\mathrm{EPOCH}$ values. These models are simplified and described as shown in Table 2. An optimized image algorithm for CNN prediction and conditional random field (CRF) algorithm were used to conduct post-processing of SegNet model prediction in this study. 
Table 2 Description of the SegNet models

\begin{tabular}{|c|c|}
\hline Models & Description \\
\hline model-a & Single-class SegNet model of karst aquatic flora with EPOCH 5,10 or 15 \\
\hline model-b & Single-class SegNet model of broad-leaved forest with EPOCH 5,10 or 15 \\
\hline model-g & Single-class SegNet model of karst grass with EPOCH 5,10 or 15 \\
\hline model-w & Single-class SegNet model of karst water with EPOCH 5,10 or 15 \\
\hline multi-class SegNet model & the multi-classification models with EPOCH 5,10 or 15 \\
\hline \multirow{3}{*}{$\begin{array}{l}\text { multi-class SegNet model texture feature } \\
\text { fusion of single-class SegNet model texture feature }\end{array}$} & the multi-class SegNet model with texture feature and EPOCH 10 or 15 \\
\hline & the fusion of four single-class SegNet models with texture feature and EPOCH \\
\hline & 10 or 15 \\
\hline
\end{tabular}

\subsubsection{Creating Training Dataset for SegNet Model}

This study attempted to explore the impact of texture features on the SegNet model for classifying karst wetland vegetation. The deep-learning image dataset of karst wetland (Area B) was added texture features from UAV DOM image. The textural feature was calculated from the greylevel co-occurrence matrix (GLCM) with window size $3 \times 3,5 \times 5$, and $7 \times 7$ and 64 greyscale quantization levels to generate the mean, variance, homogeneity, contrast, dissimilarity, entropy, second moment and correlation features in ENVI 5.4 software, respectively. The deep-learning image dataset of karst wetland (Areas A and B) is randomly divided into 100,000 image datasets with the size of $256^{*} 256$ pixels, of which $75 \%$ was training datasets and $25 \%$ was testing datasets. The data enhancement operation process was as follows: (1) The image and label are randomly flipped along the $\mathrm{x}$-axis or $\mathrm{y}$-axis mirrored or rotated $90^{\circ}, 180^{\circ}, 270^{\circ}$ randomly; (2) the image is color shifted; (3) the image and label are randomly scaled; (4) the image is processed random invert operation.

To evaluate the performance of the multi-class SegNet model and the single-class SegNet model for discriminating karst wetland vegetation. This study made four single-class training datasets for karst water, karst grass, karst broad-leaved forest, and karst aquatic flora, respectively. The paper also produced two multi-class training datasets including four karst vegetation types.

\subsubsection{SegNet Model Training and Parameter Optimization}

Training model mainly includes parameter setting and self-optimization of the model during training. Before training model, the operation is conducted to set the training parameters, and then input a large number of reprocessed training samples into the CNN network. A large number of nonlinear transformation combinations were performed on the data according to the network to obtain high-level abstract features and transfer them to the output layer. Then calculate the difference between the output data and the actual data. Then optimize the weight matrix based on this difference so that the difference between the output data and the actual data can be minimized, to achieve the purpose of optimizing the model.

Training model in this study was performed on a desktop terminal configured with an NVIDIA GeForce GTX 1080 8G and an Intel Core i7 8700K. Before training model, we set the main parameters of the SegNet model. The learning rate can control the learning progress of the model. If it is too small that it will cause the model to converge slowly, and if it is too large that it would cause divergence. The initial value of learning rate in this study was set to 0.001 . Gamma can control the rate of change of the learning rate, which was set to 0.1 in this study. The momentum plays a role in accelerating convergence. In this study, the momentum parameter was set to 0.8 . Weight decay can adjust the impact of model complexity on the loss function. The weight decay is set to 0.0001 . The step size value is set to 1000 times. Due to the limited performance of currently used desktop terminals and 100,000 training image datasets, the batch-size is set to 8 . To achieve better results for model classification, 
according to the previous research of Zhang et al.[49] and Ruder [50], this paper sets Optimizer to SGD and Loss Function to category-cross-entropy loss, respectively. This study wants to explore the influence of EPOCH value on the SegNet model for discriminating karst wetland vegetation. This paper conducted SegNet models training for karst wetland of Area A using the different EPOCH values from 5 to 15. The SegNet models training for karst wetland of Area B set the EPOCH values from 5 to 10 .

\subsubsection{Optimized Image Algorithm for SegNet Prediction}

In this study, the SegNet model classified karst wetland vegetation using the segmented subimage with the size of $256^{*} 256$ pixels. Therefore, the classification results of each sub-image were needed to mosaic into the final result. The trimming image predicts that re-stitching resulted in relatively obvious stitching marks in the final result, which significantly reduced the model classification accuracy.

To solve this problem, this study designed an optimized image algorithm for post-processing of SegNet-based classifications to eliminate the stitching traces. The optimized image algorithm integrated edge detection method with filtering algorithm. To eliminate the artifacts generated by edge detection, this method used image edge mirror expansion. The core idea of optimizing image algorithms is to expand the range of predicted images, then crop the images after prediction, and finally splice them into model predicted images. The specific steps of this method were as follows: (1)When the SegNet model conducted prediction, the UAV image was cropped into sub-images with a size of $128 * 128$ pixels. (2)Then expand the size of sub-images from $128 * 128$ pixels to $256 * 256$ pixels through the mirror operation. (3)The SegNet model predicted the sub-images with a size of $256 * 256$ pixels. (4)The predicted sub-image with the size of $256 * 256$ pixels was then trimmed into an image with a size of $128 * 128$ pixels. Finally, the corresponding position of (1) was stitched on the prediction sub-image. (5)Finally repeat the step (1)-(4) until the SegNet model completely predicted the UAV images of Area A and B. The processing of optimizing image algorithm was shown in Figure 4

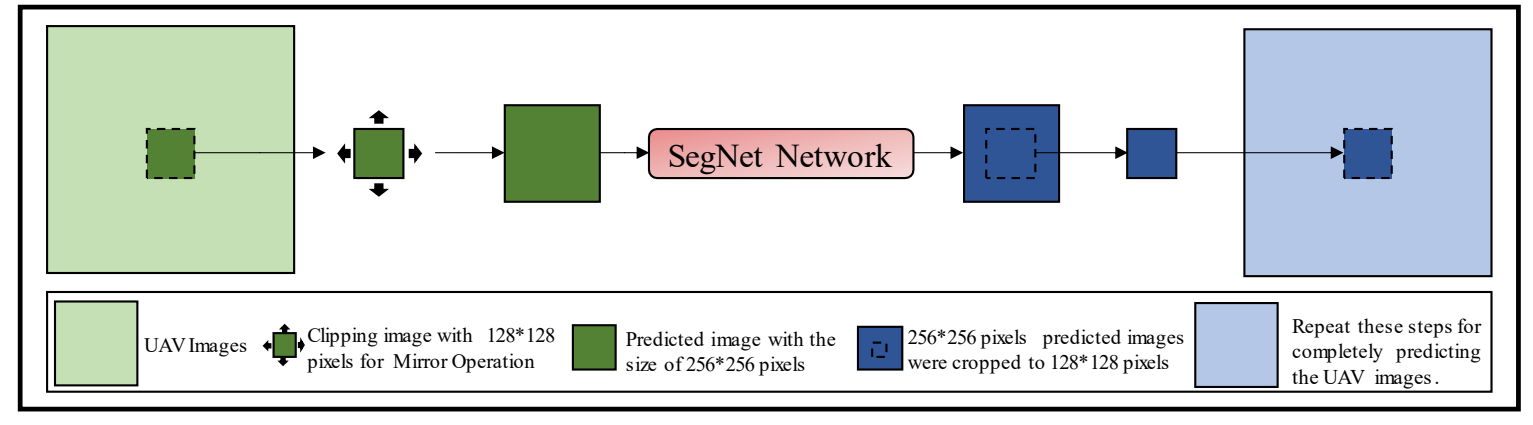

Figure 4 Processing of optimizing image algorithm for post-processing of SegNet model prediction

\subsubsection{Fusion Algorithm of Single-class SegNet Models}

This study aimed to compare multi-class SegNet models and fusion of single-class SegNet models, and find an optimal model for classifying karst wetland vegetation. The method of maximum probability is adopted as a method for integrating four single-class SegNet classifications into a multiclass SegNet classification. The fusion algorithm utilized the Equation (1) to calculated each singleclass SegNet model $\left(M_{j}, j=1,2 \ldots 4\right)$ prediction probability $\left(P_{i j}\left(X_{i}\right)\right)$ of four vegetation types $\left(X_{i}, i=1,2 \cdots 4\right)$ at each pixel, and summarized of the probability of each vegetation $\left(P\left(X_{i}\right)\right)$ type at a pixel. Finally, the method selected the vegetation type of maximal probability at each pixel as the final multi-class SegNet classification results. The specific fusion procedure was shown in Figure 5. 


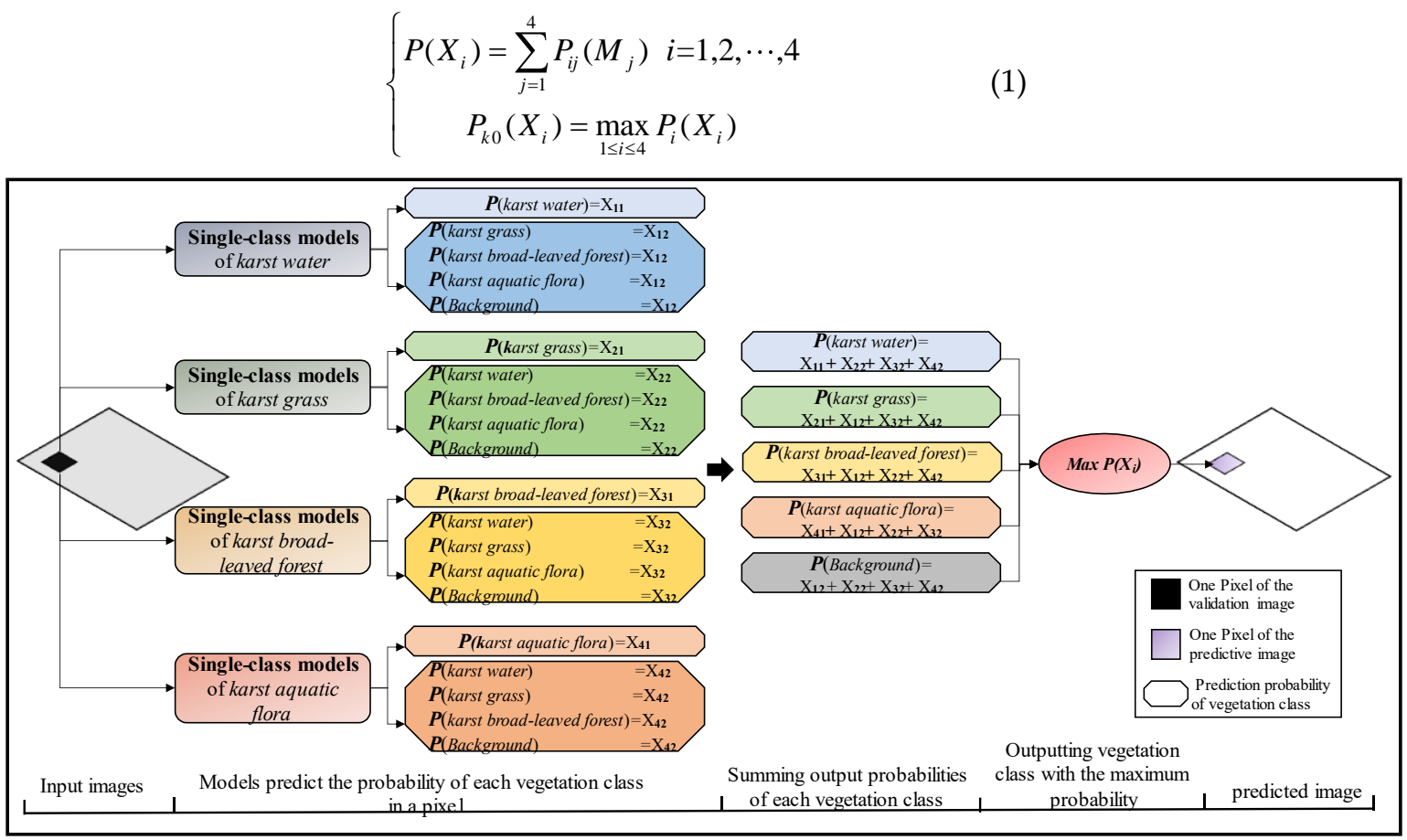

Figure 5 The fusion procedure of single-class SegNet Models

\section{Results Analysis and Accurate Evaluation}

An error matrix, Kappa coefficient, overall accuracy, Recall, F1-score and class-specific producer's and user's accuracies were reported for single-class SegNet classification, multi-class SegNet classification and fusion of single-class SegNet classification in this paper using the testing sample dataset and semantic labeling dataset.

\subsection{Single-class SegNet Models with EPOCH}

To explore the effect of different EPOCH values on single-class SegNet Models for discriminating karst wetland vegetation. This paper developed four single-class SegNet models with the different EPOCH values $(5,10,15)$. The classifications of each model were shown in Figure 6 . The red circle highlighted the mis-classification of karst vegetation type. Comparison of semantic labeling dataset in the white circle, this study found that (1) the model-w with all EPOCH values $(5,10,15)$ can produce good classification results for karst water, and only the isolated trees in the small lakes was falsely identified as karst water. In general, with the increase of EPOCH values, the classification of model-w for karst water is better. (2) Several area of karst grass was not identified using the model-g. The classification results of model-g with EPCOH 15 were better than the models with the other two EPCOH values. (3) The model-b with three EPCOH values was able to classify karst broad-leaved forest, but the whole area of karst broad-leaved forest was not completely extracted. (4) The model-a with three $\mathrm{EPCOH}$ values well depicted aquatic flora vegetation in study area, but the small area of aquatic flora vegetation was not identified. This study found that the single-class SegNet model with EPCOH 15 produced more visually accurate depictions of karst wetland vegetation. 


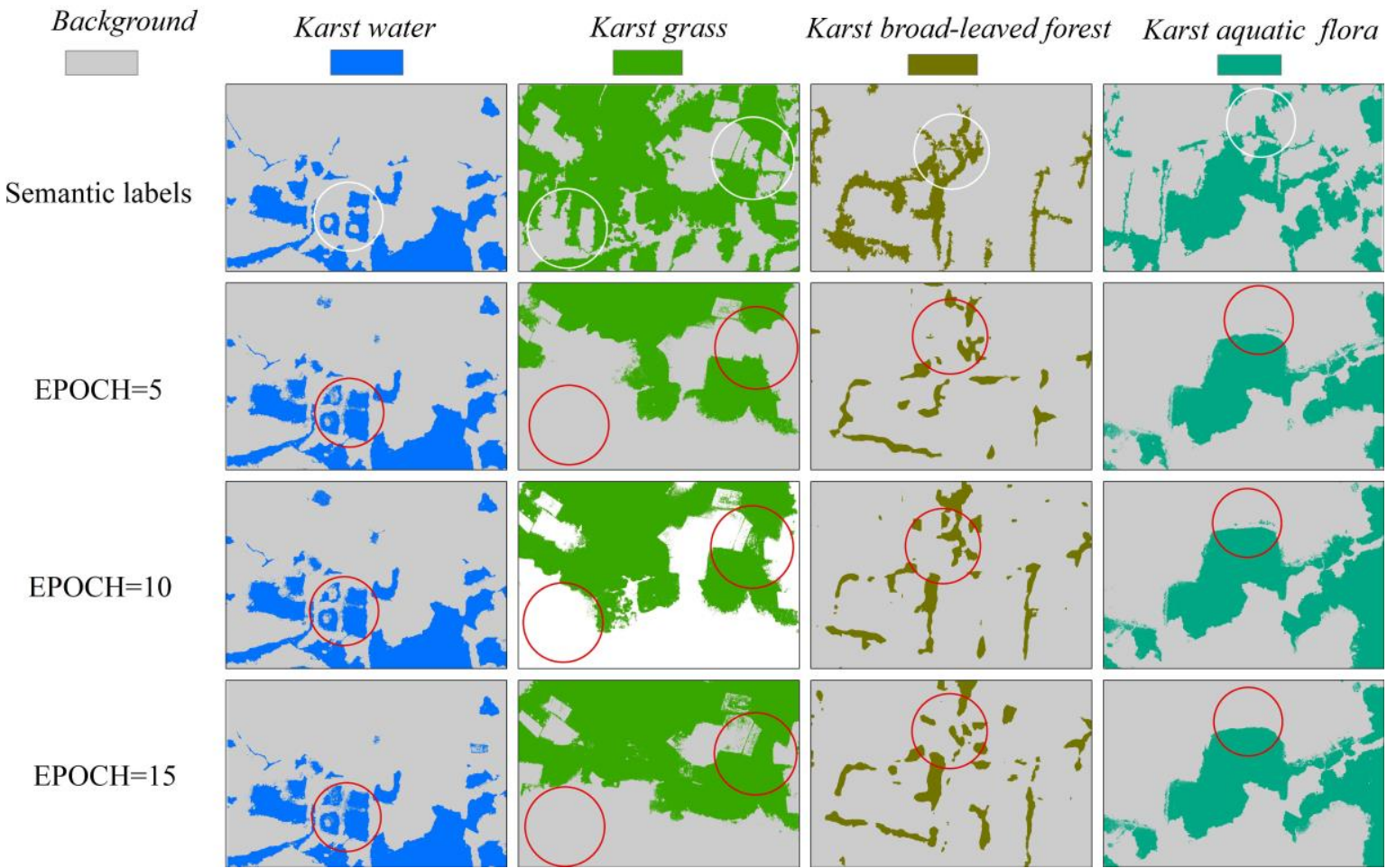

Figure 6 Classifications using single-class SegNet models with different EPOCH values

Table 3 The classification accuracies of single-class SegNet models for karst wetland with different EPOCH values based on testing data

\begin{tabular}{|c|c|c|c|c|c|c|}
\hline Models & Karst vegetation & EPOCH & Precision & Recall & F1-score & Kappa \\
\hline \multirow{3}{*}{ model-a } & \multirow{3}{*}{ Karst Water } & 5 & 0.92 & 0.93 & 0.92 & 0.85 \\
\hline & & 10 & 0.92 & 0.97 & 0.94 & 0.88 \\
\hline & & 15 & 0.93 & 0.98 & 0.96 & 0.91 \\
\hline \multirow{3}{*}{ model- $b$} & \multirow{3}{*}{ Karst Grass } & 5 & 0.77 & 0.74 & 0.75 & 0.50 \\
\hline & & 10 & 0.86 & 0.74 & 0.78 & 0.56 \\
\hline & & 15 & 0.84 & 0.76 & 0.79 & 0.58 \\
\hline \multirow{3}{*}{ model-g } & \multirow{3}{*}{$\begin{array}{c}\text { Karst Broad-leaved } \\
\text { Forest }\end{array}$} & 5 & 0.93 & 0.70 & 0.75 & 0.52 \\
\hline & & 10 & 0.94 & 0.72 & 0.78 & 0.56 \\
\hline & & 15 & 0.95 & 0.78 & 0.83 & 0.66 \\
\hline \multirow{3}{*}{ model-w } & \multirow{3}{*}{ Karst Aquatic Flora } & 5 & 0.81 & 0.89 & 0.84 & 0.67 \\
\hline & & 10 & 0.83 & 0.89 & 0.85 & 0.69 \\
\hline & & 15 & 0.85 & 0.91 & 0.87 & 0.74 \\
\hline
\end{tabular}

Table 3 displays the classification accuracies for four single-class SegNet models, which indicated that the modes with EPOCH 15 was able to achieve higher classification accuracy. The single-class SegNet models achieved over 75\% F1-score for all vegetation classes, but the kappa coefficient of karst grass was below $60 \%$. The model-w achieved the highest classification accuracy (F1-score, 0.96) when utilizing DOM, followed by model-w (F1-score,0.87) and model-g (F1-score,0.83). The karst Grass and karst Broad-leaved Forest were difficult to discern in the single-class SegNet classifications. Karst water was accurately classified in the model- $w$ classification achieving 0.91 kappa coefficient. The model-g with EPOCH 15 improved F1-score to 0.83 for Karst Broad-leaved Forest, an increase of $8 \%$ in comparison of using EPOCH 5 or 10 . The conclusion in this study was confirmed 
that the all single-class SegNet models improved above 7\% of kappa coefficient when using the EPOCH 15, especially for the model-g, which improved $14 \%$. It is demonstrated that the EPOCH values affected the single-class SegNet model for karst vegetation classifications.

\subsection{Multi-class SegNet Models with EPOCH}

Figure 7 shows the karst vegetation classifications of Area A produced by fusion of single-class SegNet model and the multi-class SegNet model with three EPOCH values, respectively. The classification results of each model in the Figure 9 are marked with more observable errors in the red circles, and the correct vegetation type at the corresponding position is marked with white circles.

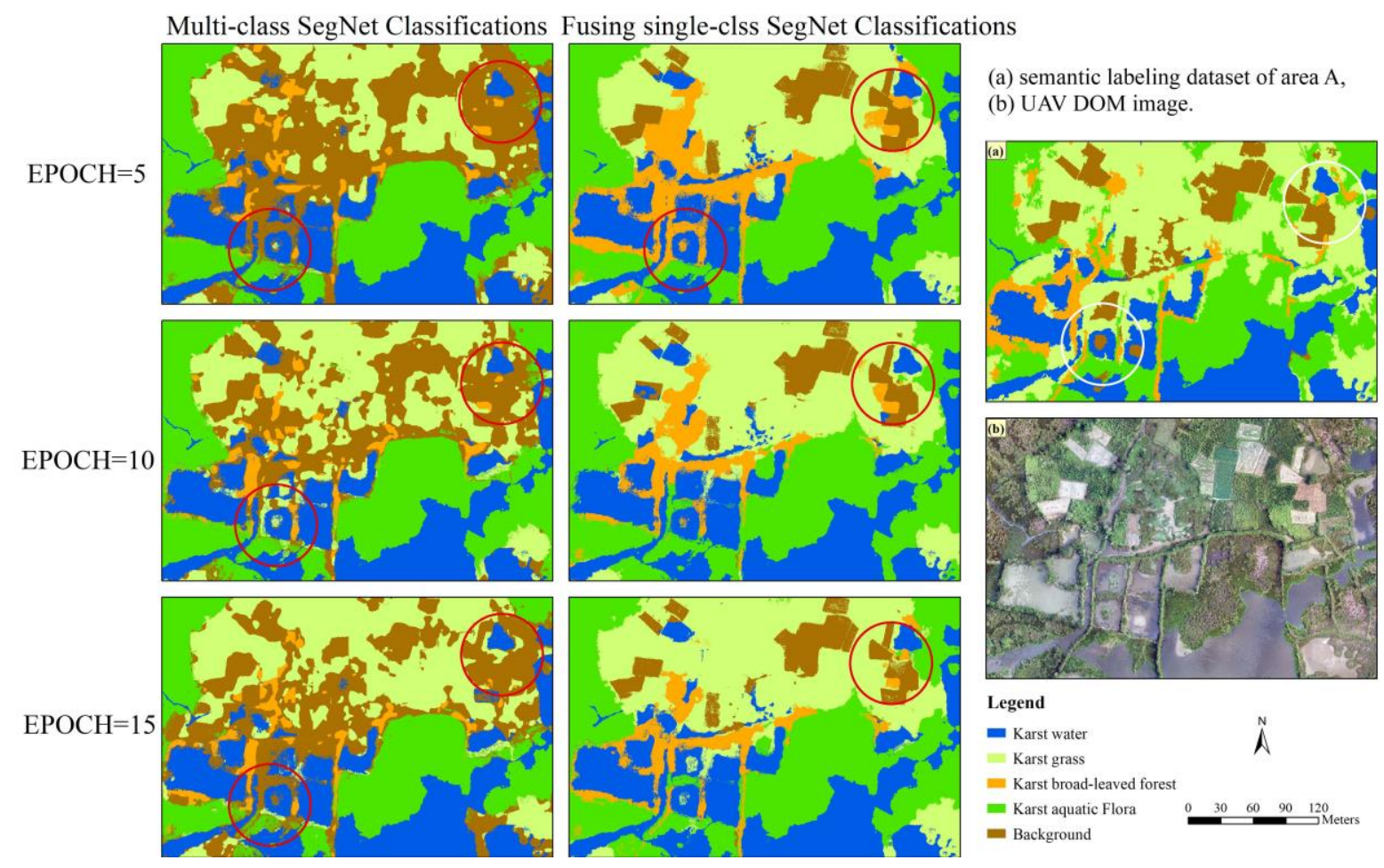

Figure 7 Results of SegNet-based classifications for all karst wetland types with EPOCH

Figure 9 shows that the classification results of the two models become better with the increase EPOCH value. we found that karst broad-leaved forest and karst Grass are better depicted by fusion of single-class SegNet model than by multi-class SegNet model in specific areas. For example, the predominance of karst broad-leaved forest of the study area was better represented around the karst river and ponds by single-class SegNet model without confusing between karst Grass and karst Aquatic Flora. Karst water and karst Aquatic Flora were both better depicted using the SegNet models. Through visual comparison of classifications derived from multi-class SegNet model and fusion of single-class SegNet model, the major visual difference in classifications is the other land use types, such as paddy field and vegetable fields in the study area, which were depicted mixed with karst Grass due to the spectral similarity. 
Table 4 The classification accuracies of SegNet models with different EPOCH values based on testing data

\begin{tabular}{|c|c|c|c|c|c|c|}
\hline \multirow[b]{2}{*}{ Models } & \multirow[b]{2}{*}{ EPOCH } & \multicolumn{4}{|c|}{ F1-Score } & \multirow[b]{2}{*}{ Kappa } \\
\hline & & Karst Water & Karst Grass & $\begin{array}{l}\text { Karst Broad- } \\
\text { leaved Forest }\end{array}$ & $\begin{array}{c}\text { Karst Aquatic } \\
\text { Flora }\end{array}$ & \\
\hline \multirow{3}{*}{$\begin{array}{c}\text { Multi-class SegNet } \\
\text { Model }\end{array}$} & 5 & 0.88 & 0.61 & 0.46 & 0.71 & 0.59 \\
\hline & 10 & 0.86 & 0.63 & 0.50 & 0.69 & 0.63 \\
\hline & 15 & 0.91 & 0.68 & 0.52 & 0.75 & 0.68 \\
\hline \multirow{3}{*}{$\begin{array}{c}\text { Fusion of single- } \\
\text { class SegNet } \\
\text { model }\end{array}$} & 5 & 0.91 & 0.59 & 0.52 & 0.80 & 0.65 \\
\hline & 10 & 0.89 & 0.67 & 0.65 & 0.75 & 0.70 \\
\hline & 15 & 0.92 & 0.66 & 0.66 & 0.81 & 0.74 \\
\hline
\end{tabular}

Table 4 displays the classification accuracies(F1-score) for fusion of single-class SegNet model and multi-class SegNet model. The fusion of single-class SegNet model obtained higher F1-score and kappa coefficient than multi-class SegNet model for all karst vegetation classifications, with the exception of karst grass. Karst water both achieved over 0.86 F1-score using the two multi-class models. When EPOCH value increased from 5 to 15, the kappa coefficient of fusion of single-class SegNet model and multi-class SegNet model both improved 0.09, especially for fusion of single-class SegNet model increasing from 0.65 to 0.74 . Meanwhile, the fusion of single-class SegNet model promote more classification accuracy (F1-score) for each karst wetland vegetation compared to multiclass SegNet model. The SegNet models with EPOCH 5 for karst aquatic flora achieved higher F1score than the model with EPOCH 10. The fusion of single-class SegNet model with EPOCH 510 for karst grass achieved the highest F1-score than the other EPOCH values. Those conclusions indicated that the iterative times of SegNet model affected the classification accuracy of karst wetland vegetation. When comparing single-class SegNet-based classifications (such as the model-a, model-b), the multi-class SegNet classifications achieved the smaller F1-scoers for each vegetation type, but the difference in kappa coefficient was not statistically significant. Those conclusions demonstrated that not only the fusion of single-class SegNet model was more accurate in classifying karst wetland vegetation, but also the SegNet model with EPOCH 15 also outperformed the models with EPOCH 5 or 10 .

\subsection{Single-class SegNet Models with Texture Feature}

This study added textural features into the deep-learning image dataset of Area B for exploring the impact of texture feature on single-class SegNet classifications of karst wetland vegetation (Figure 8). In order to comparison of visual difference in classifications, the typical area of each model classification was marked with red circles, and the correct vegetation type at the corresponding position was marked with a white circle from semantic labeling dataset. 


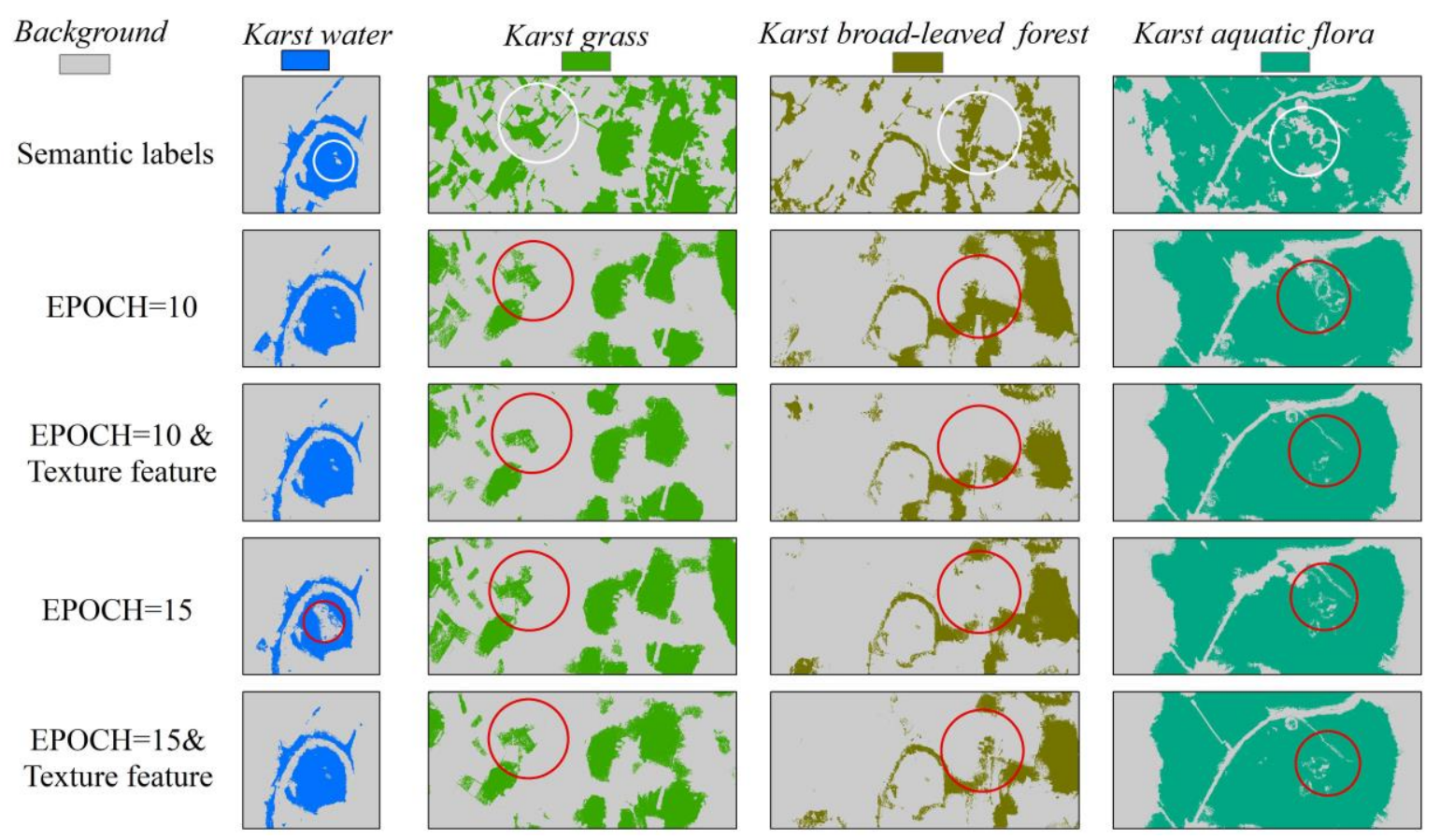

Figure 8 Results of single-class SegNet-based classifications for all karst wetland types with EPOCH and textural features

The single-class SegNet model produced more visually accurate depictions of karst water and karst aquatic flora than karst grass and karst broad-leaved forest. The single-class SegNet model with EPOCH 15 and textural features accurately identified four karst vegetation. When the EPOCH value was set 10 , the four models without textural features produced more visually accurate depictions than that with textural features. While the four models with textural features and EPOCH 15 provided the better depictions. Comparison of the models with texture features, the karst vegetation classifications were better depicted by the model with the EPOCH 15 than by the model with the EPOCH 10. The model- $a$ with the EPOCH 15 produced the confusion between karst water and other land use types. The model- $g$ with the EPOCH 10 produced the better depiction of karst broad-leaved forest than the model with EPOCH 15 or texture features (Figure 8). 
Table 5 The classification accuracies of single-class SegNet models with texture feature and different EPOCH values in areas B based on testing data

\begin{tabular}{|c|c|c|c|c|c|c|c|}
\hline Models & karst vegetation & $\begin{array}{l}\text { Textural } \\
\text { Feature }\end{array}$ & Epoch & Precision & Recall & F1-score & Kappa \\
\hline \multirow{4}{*}{ model-a } & \multirow{4}{*}{ Karst Water } & \multirow{2}{*}{$\mathrm{NO}$} & 10 & 0.95 & 0.92 & 0.91 & 0.87 \\
\hline & & & 15 & 0.98 & 0.93 & 0.95 & 0.90 \\
\hline & & \multirow{2}{*}{ YES } & 10 & 0.95 & 0.92 & 0.94 & 0.87 \\
\hline & & & 15 & 0.97 & 0.97 & 0.97 & 0.94 \\
\hline \multirow{4}{*}{ model- $b$} & \multirow{4}{*}{ Karst Grass } & \multirow{2}{*}{$\mathrm{NO}$} & 10 & 0.81 & 0.87 & 0.83 & 0.66 \\
\hline & & & 15 & 0.83 & 0.90 & 0.86 & 0.72 \\
\hline & & \multirow{2}{*}{ YES } & 10 & 0.85 & 0.89 & 0.87 & 0.72 \\
\hline & & & 15 & 0.87 & 0.89 & 0.88 & 0.78 \\
\hline \multirow{4}{*}{ model-g } & \multirow{4}{*}{$\begin{array}{l}\text { Karst Broad- } \\
\text { leaved Forest }\end{array}$} & \multirow{2}{*}{ NO } & 10 & 0.92 & 0.82 & 0.86 & 0.72 \\
\hline & & & 15 & 0.94 & 0.84 & 0.87 & 0.77 \\
\hline & & \multirow{2}{*}{ YES } & 10 & 0.92 & 0.82 & 0.86 & 0.74 \\
\hline & & & 15 & 0.95 & 0.89 & 0.92 & 0.83 \\
\hline \multirow{4}{*}{ model-w } & \multirow{4}{*}{$\begin{array}{c}\text { Karst Aquatic } \\
\text { Flora }\end{array}$} & \multirow{2}{*}{$\mathrm{NO}$} & 10 & 0.91 & 0.86 & 0.88 & 0.76 \\
\hline & & & 15 & 0.93 & 0.91 & 0.92 & 0.82 \\
\hline & & \multirow{2}{*}{ YES } & 10 & 0.93 & 0.91 & 0.92 & 0.84 \\
\hline & & & 15 & 0.96 & 0.94 & 0.95 & 0.90 \\
\hline
\end{tabular}

Table 5 displays the classification accuracies for four single-class SegNet models with or without texture feature in the area B. The single-class SegNet models for classifying karst vegetations achieved over $83 \%$ of F1-score, especially for the models with the textural features producing $0.97 \mathrm{~F} 1$-score and 0.94 kappa coefficient for karst water. These results indicated that the single-class SegNet model provides the capability for monitoring spatial distribution of karst wetland vegetation using UAV images. The four models with the textural features improved the $4 \%$ F1-score and $6 \%$ kappa coefficient when comparing the model without texture feature classifications, respectively. The studies found that the classifications produced by combining single-class SegNet with texture features typically outperform only SegNet-based classifications. The texture feature enhances the SegNet model's capabilities in discriminating karst vegetations, and achieved over 0.92 F1-score for karst water, karst broad-leaved forest and karst aquatic flora. When the deep-learning dataset without texture feature, the SegNet models with EPOCH 15 achieved over 0.92 F1-score for karst water and karst aquatic flora, which was higher than the other two karst vegetation types. When comparing the model classifications with the different EPOCH values, this paper found the following results: (1) the model- $a$ with the EPOCH 15 and texture features produced 0.97 F1-score and 0.94 kappa coefficient for karst water, which was over 0.06 and 0.07 than the model with both EPOCH 10 and without texture features. the model- $a$ using just EPOCH 10 achieved 0.90 kappa coefficient, a decrease of $4 \%$ in comparison of the model with both EPOCH 15 and texture features;(2) the model- $b$ with the EPOCH 15 improved F1-score and kappa coefficient to 0.86 and 0.72 comparison of the model with the EPOCH 10, while using the combination of EPOCH 15 and texture features the model- $b$ achieved higher classification accuracy for karst grass, reaching 0.88 F1-score and 0.78 kappa coefficient, an increase of $12 \%$ in comparison of the model with the just EPOCH 10;(3) the model-g classifications with the EPOCH 15 and 10 both achieved over 0.86 F1-score for karst broad-leaved forest. This corresponds with the use of the combination of EPOCH 10 and texture features. The model-g using the combination of EPOCH 15 and texture features improved F1-score and kappa coefficient to 0.92 and 0.83 , an increase of $6 \%$ and $11 \%$ in comparison of the model with just the EPOCH 10 , respectively; 
(4) the model-w with EPOCH 15 achieved higher classification accuracy for karst aquatic flora than the model with EPOCH 10, reaching 0.92 F1-score and 0.82 kappa coefficient. There were not differences in classification accuracy between the model-w with EPOCH 10 and the model-w with the combination of EPOCH 10 and texture features. The model-w using the combination of EPOCH 15 and texture features improved F1-score and kappa coefficient to 0.95 and 0.90 , an increase of $7 \%$ and $14 \%$ in comparison of the model with just the EPOCH 10, respectively. These results indicated that the single-class SegNet model is able to improve classification accuracy for karst vegetation types by using the combination of 10 and texture features.

\subsection{Multi-class SegNet Models with Texture Feature}

This paper attempted to evaluate the influence of texture features in discriminating karst wetland vegetation between the multi-class SegNet model and fusion of single-class SegNet models in area B. In this study, four single-class SegNet models with the same EPOCH value were fused into multi-class SegNet classifications. The classification results were shown in Figure 9. In order to comparison of visual difference in classifications, the typical area of each model classification was marked with red circles, and the correct vegetation type at the corresponding position was marked with a white circle from semantic labeling dataset.

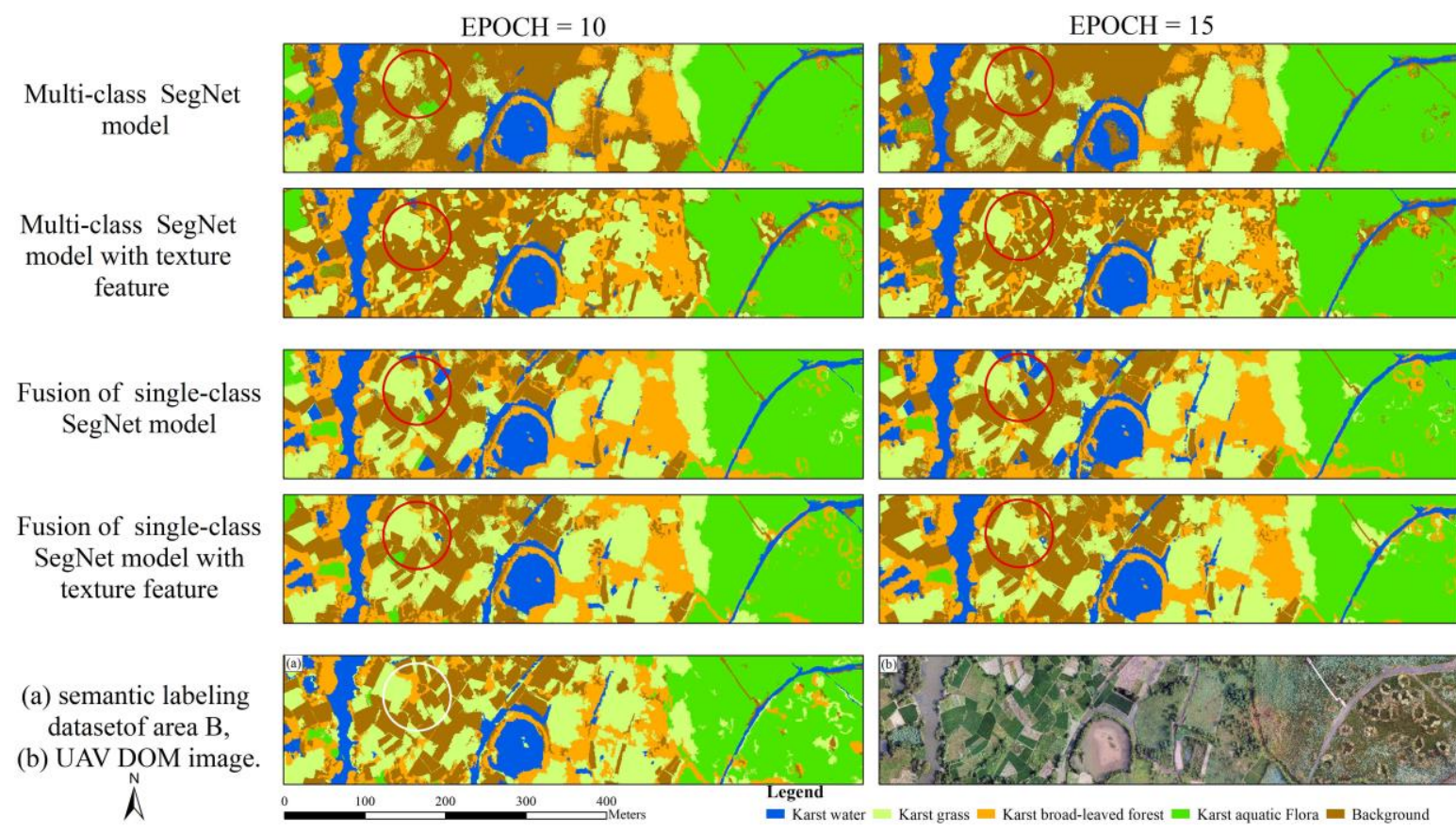

Figure 9 Results of multi-classification SegNet models with texture feature

The two multi-class SegNet-based classifications seen from Figure 11 produced more visually accurate depictions of karst water and karst aquatic flora than karst grass and karst broad-leaved forest in the area B. The two multi-class SegNet models both overestimated classification results for karst broad-leaved forest, while the models underestimated classification of karst grass (Figure 9). The multi-class SegNet model with EPOCH 10 produced more visually accurate depictions of karst water and karst grass than that with EPOCH 15. This corresponds with the use of the combination of $\mathrm{EPOCH}$ values and texture features. When using the combinations of $\mathrm{EPOCH}$ values and texture features, the SegNet model accurately identified karst vegetation, especially for the karst broadleaved forest, comparing the model without texture features. Comparison of the two multi-class SegNet model without texture features, the classifications of karst grass with texture features produced by the SegNet model have much more small patches. For classifications based on multiclass SegNet model without texture features, the eastern of the study area in the river valley depicts mixed karst aquatic flora with karst broad-leaved forest and other land cover types due to the spectral 
similarity. There were not visual differences in classifications produced by fusion of single-class SegNet models with the EPOCH values between 15 and 10. When comparing the fusion of singleclass model classifications, this paper found that the models with texture features produced more visually accurate depictions than that without textural features, especially for karst grass and karst broad-leaved forest. Through visual comparison of multi-class SegNet-based classifications using the texture features and fusion of single-class SegNet classifications, we found that karst grass and karst broad-leaved forest are better depicted by multi-class SegNet-based classifications than by fusion of single-class SegNet classifications in specific areas. Whereas when the fusion of single-class SegNet model using texture features, the fused SegNet-based classifications provided the better depictions of four karst vegetations than multi-class SegNet-based classifications using the texture features.

Table 6 The classification accuracies of multi-class SegNet models with texture features and different EPOCH values based on testing data

\begin{tabular}{|c|c|c|c|c|c|c|c|}
\hline \multirow[b]{2}{*}{ Models } & \multirow[b]{2}{*}{$\begin{array}{l}\text { Textural } \\
\text { Feature }\end{array}$} & \multirow[b]{2}{*}{ EPOCH } & \multicolumn{4}{|c|}{ F1-Score } & \multirow[b]{2}{*}{ Kappa } \\
\hline & & & Karst Water & Karst Grass & $\begin{array}{c}\text { Karst Broad- } \\
\text { leaved } \\
\text { Forest }\end{array}$ & $\begin{array}{c}\text { Karst } \\
\text { Aquatic } \\
\text { Flora }\end{array}$ & \\
\hline \multirow{4}{*}{$\begin{array}{c}\text { Multi-class } \\
\text { SegNet model }\end{array}$} & \multirow{2}{*}{ NO } & 10 & 0.89 & 0.71 & 0.71 & 0.78 & 0.70 \\
\hline & & 15 & 0.90 & 0.78 & 0.73 & 0.82 & 0.73 \\
\hline & \multirow{2}{*}{ YES } & 10 & 0.89 & 0.79 & 0.76 & 0.87 & 0.75 \\
\hline & & 15 & 0.92 & 0.81 & 0.86 & 0.84 & 0.76 \\
\hline \multirow{4}{*}{$\begin{array}{l}\text { Fusion single- } \\
\text { class SegNet } \\
\text { model }\end{array}$} & \multirow{2}{*}{ NO } & 10 & 0.95 & 0.76 & 0.76 & 0.81 & 0.76 \\
\hline & & 15 & 0.95 & 0.77 & 0.73 & 0.87 & 0.81 \\
\hline & \multirow{2}{*}{ YES } & 10 & 0.97 & 0.77 & 0.81 & 0.81 & 0.81 \\
\hline & & 15 & 0.97 & 0.79 & 0.83 & 0.83 & 0.84 \\
\hline
\end{tabular}

For multi-class SegNet-based classifications of karst vegetation in area B, the model with EPOCH 15 and 10 both achieved over 70\% F1-score and kappa coefficient for all vegetation classes (Table 6). Karst water was accurately classified in the model with the EPOCH 15 achieving 0.90 F1score and, which is higher than the other karst vegetation. The SegNet-based classifications using the EPOCH 15 for karst broad-leaved forest improved F1-score to 0.82 , an increase $4 \%$ of in comparison of the model with the EPOCH 10. The SegNet-based classifications for karst grass with the EPOCH 15 produced 0.78 F1-score, which was over 0.07 than the classifications with the EPOCH 10 . When using the combinations of EPOCH values and texture features, the model produced the higher classification accuracy than the model just with the EPOCH. The multi-class SegNet-based classifications using the combination of the EPOCH 15 and texture features achieved over $0.81 \mathrm{~F} 1-$ score for all vegetation classes. The model achieved the highest classification accuracy for karst water (F1-score, 0.92) when both utilizing the EPOCH 15 and texture features, followed by karst broadleaved forest (F1-score, 0.86). The SegNet-based classifications using combinations of EPOCH 10 and texture features for karst broad-leaved forest improved F1-score to 0.86 , an increase $10 \%$ of in comparison of the model with the EPOCH 10. The SegNet-based classifications for karst aquatic flora with the combinations of EPOCH 10 and texture features produced higher accuracy, achieving 0.87 F1-score when comparing to the model using the combinations of EPOCH 15 and texture features. There were not differences in classification accuracy produced by the model between just with $\mathrm{EPOCH} 15$ and with the combination of EPOCH 10 and texture features. The model using the combination of EPOCH 15 and texture features improved kappa coefficient to 0.76 , an increase of $6 \%$ in comparison of the model with just the EPOCH 10. Meanwhile, the model with textural features improved 8\% F1-score between in all karst vegetation classes when compared to the model with just the EPOCH 10, especially for karst broad-leaved forest. 
For the classifications of karst vegetation using the fused single-class SegNet models in area B, the model with EPOCH 15 and 10 both achieved over 0.71 F1-score and 0.76 kappa coefficient for all vegetation classes. There were not differences in classification accuracy produced by the models with the EPOCH values between 10 and 15. The SegNet-based classifications for karst water produced 0.95 F1-score, which was higher than the other vegetation types. The classifications using the EPOCH 15 improved kappa coefficient to 0.87 , an increase of $6 \%$ in comparison of the model using the EPOCH 10. When using the combinations of EPOCH values and texture features, the model produced the higher classification accuracy than the model just with the EPOCH for all vegetation classes, except for karst aquatic flora. The fused SegNet-based classifications using the combination of the EPOCH 15 and texture features achieved over 0.79 F1-score for all vegetation classes. The fused model achieved the highest classification accuracy for karst water (F1-score, 0.97) when utilizing the combination of the EPOCH 10 and texture features (Table 6). There were not differences in classification accuracy produced by the fused model with the combination of texture features and the EPOCH values increasing from 10 to 15. The fused model with the EPOCH 15 achieved 0.87 F1-score for karst aquatic flora, while the fused model with the combination of texture features achieved 0.81 F1-score for this class. The fused model using the combination of EPOCH 15 and texture features improved 8\% kappa coefficient when compared to the model with just the EPOCH 10.

Through comparison of multi-class SegNet-based and fused SegNet-based classifications in area B, this study found that the multi-class SegNet model produced the higher F1-score than the fused model for all vegetation classes, except for karst water. Whereas the fused model improved kappa coefficient to 0.84 , an increase $14 \%$ of in comparison of the multi-class model with EPOCH 10.

\subsection{Comparison of RF classifications and fusion of single-class SegNet classifications}

This paper found that the fusion of single-class SegNet model using the combination of EPOCH 10 and texture features provides the best performance in mapping karst vegetation. Table 7 displays the overall accuracies for the fused SegNet-based classifications and object-based RF classifications in area A and area B. There were not differences in classification accuracies between object-based RF model and the fused SegNet model, both achieving over $87 \%$ overall classification accuracy. User's and producer's accuracies were summarized for each class in four classification scenarios (Table 8). Object-based RF classifications achieved over 90\% producer's accuracy for all categories. Karst water was accurately classified by object-based RF model and the fused SegNet model achieving over 95\% user's and producer's accuracy, respectively. The object-based RF classifications achieved over $90 \%$ producer's accuracy for karst broad-leaved forest and karst aquatic flora, which is higher than the fused SegNet-based classifications. The producer's accuracy of karst grass was consistently over $90 \%$ for the two models. The two models achieved over $89 \%$ user's accuracy for all categories, with the exception of karst grass. The object-based RF classifications produced higher user's accuracy for karst grass than the fused SegNet-based classifications.

Table 7 Comparison of classification accuracy between object-based RF model and fusion of singleclass SegNet model

\begin{tabular}{|c|c|c|c|c|c|}
\hline Models & Accuracy Evaluation & Karst Water & Karst Grass & $\begin{array}{l}\text { Karst Broad- } \\
\text { leaved Forest }\end{array}$ & $\begin{array}{c}\text { Karst Aquatic } \\
\text { Flora }\end{array}$ \\
\hline \multirow{4}{*}{ Object-based RF model } & Producer Accuracy & 0.95 & 0.90 & 0.95 & 0.90 \\
\hline & User Accuracy & 0.96 & 0.94 & 0.95 & 0.89 \\
\hline & OA & \multicolumn{4}{|c|}{0.92} \\
\hline & Kappa & \multicolumn{4}{|c|}{0.90} \\
\hline \multirow{4}{*}{$\begin{array}{l}\text { Fusion of single-class } \\
\text { SegNet model }\end{array}$} & Producer Accuracy & 0.95 & 0.95 & 0.75 & 0.75 \\
\hline & User Accuracy & 1.00 & 0.68 & 0.94 & 0.94 \\
\hline & $\mathrm{OA}$ & \multicolumn{4}{|c|}{0.87} \\
\hline & Kappa & \multicolumn{4}{|c|}{0.84} \\
\hline
\end{tabular}




\section{Discussion}

The classifications using the single-class SegNet model achieved over 0.83 F1-score, and the fused SegNet-based classifications achieved $87 \%$ overall accuracy for all karst vegetation classes. The results demonstrated that the SegNet deep-learning algorithm is able to provide the high-precision classifications for mapping karst wetland vegetation using the high spatial resolution UAV images. In comparison of classification accuracy (F1-score values), there was between 5\%-21\% improvement when using the single-class SegNet-based algorithm over multi SegNet-based algorithm, and the single-class SegNet models produced over 0.88 F1-score for karst wetland vegetation, this was consistent with reported studies that the deep-learning algorithm can accurately identify simple and pure ground objects, such as river[51] and artificial building[52]. The findings in this study filled the comparison of karst vegetation classifications between single-class SegNet model and multi-class SegNet model, and could be also extended the application of SegNet in complex wetland vegetation mapping. This study found that there were not differences in classifying karst vegetation using object-based RF model and the fused single-class SegNet model with the combinations of EPOCH 10 and texture features. Other studies have found the different conclusion using the other optical data. For example, Pouliot et al.[53] compared the CNN and pixel-based RF algorithm for wetland mapping with Landsat images, and indicated that CNN is superior to the pixel-based RF algorithm. Rezaee et al. [54] evaluated the efficiency of CNN for wetland mapping, the classification results of CNN were compared with the RF classifier. The CNN model achieved $94.82 \%$ overall classification accuracy, demonstrating an improvement of about $16 \%$ compared to the RF classifier for all land cover types. The reasons for the differences of classification accuracy were that the pixel-based RF algorithm only used parameter optimization without the feature selection. The previous studies found that object-based classification typically outperform pixel-based classification when comparing overall classification accuracy for wetland using the high spatial resolution image[55]. Whereas this paper used the improved object-based RF algorithm and very high resolution UAV imagery for classifying karst vegetation, which went through a series of processes of multi-scale image segmentation, feature selection and parameter optimization. The SegNet model has been increasingly used in objects detection and image classification. However, the current problem of SegNet predictions of remote sensing images produces obvious stitching traces. In this study, we proposed an optimized image algorithm to eliminate the stitching traces of predicted images, and integrated this method and CRF algorithm into the SegNet network. The improved SegNet model produced better accuracy for classifying karst vegetation.

Model fusion or model combination is an approach to improve the performance of just single model for image classification. Zhao\& Liu [56] proposed a CNN-based feature extraction from the MINST dataset and algebraic fusion of multiple classifiers trained on different feature sets. Classifier fusion can achieve $98 \%$ classification accuracy. This paper is different from the reported study, and the single-class SegNet model was used to discriminate each karst vegetation, then the method of maximum probability was utilized to fuse single-class SegNet models for classifying all karst vegetation classes. Comparison of the accuracy differences between the fused single-class SegNet classifications and multi-class SegNet classifications, this paper found that the fused SegNet-based classifications typically outperform the direct SegNet classifications for four karst vegetation types. The fused SegNet model produced 0.81 kappa coefficient suggesting an improvement of $11 \%$ compared to the multi-class SegNet model for all karst vegetation classes. The fused SegNet model was about 20\%and 10\% more accurate than multi-class SegNet model to classify karst broad-leaved forest and karst aquatic flora, respectively. These conclusions demonstrated that the fuse SegNet model improves the performance for identifying karst vegetations using UAV images.

Increasing the number of iterations (EPOCH values) will cause loss function of the SegNet model to decrease and close to zero, and improve classification accuracy of the model. This paper investigated the effect of the SegNet model with different EPOCH values on mapping karst wetland vegetation. This study revealed that the SegNet model using the EPOCH 15 achieve higher classification accuracy (kappa coefficient) than the models with EPOCH 10 or 5. Specifically, the single-class SegNet model using the EPOCH 15 in area A was about 6\%, 8\%, 14\%, and 7\% more 
accurate than the models with EPOCH 5 to classify karst water, karst grass, karst broad-leaved forest and karst aquatic flora, respectively. The fused SegNet-based and multi-class SegNet-based classifications using the EPOCH 15 both achieved higher kappa coefficient, an increase of 9\% in comparison of the model with the EPOCH 5. This corresponds with the SegNet model using the EPOCH values between 10 and 5 in the area B. These results confirmed that the SegNet model with EPOCH 15 was more suited to karst wetland vegetation classification.

This study found that when the deep-learning image dataset added the texture features, the SegNet models improved the classification accuracy for karst vegetation, an increase of $7 \%-14 \%$ kappa coefficient comparison of the model without texture features. This was consistent with reported studies that found textural information can help improve the spectral separability of wetland vegetation[57-59].The single-class SegNet models with texture features improved over 5\% F1-score compared to the model without texture features. The multi-class SegNet model using the combination of EPOCH 10 and texture features was about 15\% F1-score more accurate than the models with EPOCH 5 to classify karst broad-leaved forest. The fused SegNet model also improved $7 \%$ F1-score for karst broad-leaved forest. This demonstrated that texture features can enhance the SegNet model to extract semantic information for identifying karst vegetation. This paper proposed and examined a good thought to solve the difficulty of producing the important semantic labeling dataset of training data for deep-learning model in the several inaccessible wetlands by integrating the object-based RF algorithm and visual interpretation.

\section{Conclusions}

Anthropogenic and natural processes have expedited karst wetland loss during the last four decades. Thus, much effort needed to preserve the diversity of species within wetland ecosystems. In this paper, the capability of state-of-the-art classification algorithm, SegNet deep-learning network, was investigated for karst wetland classification. In particular, the study examined the potential of the improved SegNet model integrated with optimized image algorithm and conditional random fields algorithm, for mapping karst wetland using UAV imagery in the Huixian National Wetland Park located in Guilin city, Guangxi province, China. We proposed a method of fusion of single-class SegNet models using the maximum probability algorithm for karst vegetation classification, and compared with object-based RF classification and multi-class SegNet classification, respectively. The study revealed that the fused SegNet-based classification outperforms multi-class SegNet-based classification when comparing classification accuracy (F1-score value). The objectbased RF classification is relative superior to the fused SegNet classification due to incorporate a series of processes of feature selection and parameter optimization. Whereas the fused SegNet model still achieved over $87 \%$ overall classification accuracy. Integrating texture feature into deep-learning image dataset can enhance the SegNet model to extract semantic information of the karst wetland vegetation. The latter observation suggests the significance of incorporating high-level spatial features and DSM into the classification scheme to reduce confusion between spectrally similar wetland classes.

Author Contributions: Conceptualization, Bolin Fu; Data curation, Man Liu; Formal analysis, Liangke Huang and Donglin Fan; Investigation, Hongchang He, Man Liu, Donglin Fan, Lilong Li , Liangke Huang and Ertao Gao; Methodology, Bolin Fu and Donglin Fan; Project administration, Bolin Fu and Hongchan He; Resources, Hongchang He; Software, Man Liu; Supervision, Donglin Fan and Lilong Li; Validation, Bolin Fu and Donglin Fan; Visualization, Bolin Fu; Writing - original draft, Bolin Fu; Writing - review \& editing, Bolin Fu.

Funding: This research was funded by the National Natural Science Foundation of China (grant no. 41801071), Natural Science Foundation of Guangxi Province (grant no. 2018GXNSFBA281015), Natural Science Foundation of Guangxi(grant no. GuikeAD20159037), the Innovation Project of Guangxi Graduate Education (grant no. YCSW2020168), the 'Ba Gui Scholars' program of the provincial government of Guangxi, and the Guilin University of Technology Foundation (grant no. GUTQDJJ2017096).

Acknowledgments: We are grateful to Guilin University of Technology, China, for providing drone equipment.

Conflicts of Interest: The authors declare that they have no known competing financial interests or personal relationships that could have appeared to influence the work reported in this paper. 


\section{References}

1. Hu, S. , Niu, Z. , Chen, Y. , Li, L. , \& Zhang, H. . (2017). Global wetlands: potential distribution, wetland loss, and status. Science of The Total Environment, 586, 319-327.

2. Meng, G. , Jing, L. , Chunlei, S. , Jiawei, X. , \& Li, W. . (2017). A review of wetland remote sensing. Sensors, 17(4), 777-.

3. Lee, T. H. (2009). A structural model to examine how destination image, attitude, and motivation affect the future behavior of tourists. Leisure sciences, 31(3), 215-236.

4. Lee, T. H., \& Hsieh, H. P. (2016). Indicators of sustainable tourism: A case study from a Taiwan's wetland.Ecological Indicators, 67, 779-787.

5. Mingwu, Z., Haijiang, J., Desuo, C., \& Chunbo, J. (2010). The comparative study on the ecological sensitivity analysis in Huixian karst wetland, China. Procedia Environmental Sciences,2, 386-398.

6. Li, Z. , Jin, Z. , \& Li, Q. . (2017). Changes in land use and their effects on soil properties in huixian karst wetland system. Polish Journal of Environmental Studies, 26(2), 699-707.

7. Jiang, X. , Xiong, Z. , Liu, H. , Liu, G. , \& Liu, W. . (2017). Distribution, source identification, and ecological risk assessment of heavy metals in wetland soils of a river-reservoir system. Environmental Science and Pollution Research, 24(1), 436-444.

8. Xu, D. , Wang, Y. , Zhang, R. , Guo, J. , Zhang, W. , \& Yu, K. . (2016). Distribution, speciation, environmental risk, and source identification of heavy metals in surface sediments from the karst aquatic environment of the lijiang river, southwest china. Environmental Science and Pollution Research, 23(9), 9122-9133.

9. Gil-Márquez, J. M., Barberá, J. A., Andreo, B., \& Mudarra, M. (2017). Hydrological and geochemical processes constraining groundwater salinity in wetland areas related to evaporitic (karst) systems. A case study from Southern Spain.Journal of Hydrology,544, 538-554.

10. Chamberlin, C. A., Bianchi, T. S., Brown, A. L., Cohen, M. J., Dong, X., Flint, M. K., ... \& Heffernan, J. B. (2019). Mass balance implies Holocene development of a low-relief karst patterned landscape. Chemical Geology,527, 118782.

11. Gao, P. , Liu, Y. , Wang, Y. , Liu, X. , Wang, Z. , \& Ma, L. Q. . (2019). Spatial and temporal changes of P and Ca distribution and fractionation in soil and sediment in a karst farmland-wetland system. Chemosphere, 220, 644-650.

12. Watts, A. C. , Watts, D. L. , Cohen, M. J. , Heffernan, J. B. , Mclaughlin, D. L. , \& Martin, J. B. , et al. (2014). Evidence of biogeomorphic patterning in a low-relief karst landscape. Earth Surface Processes and Landforms, 39(15), 2027-2037.

13. Zemeng F. , Jing L. , Tianxiang Y. , Xun Z. , Anjun L. .(2015). Scenarios of land cover in karst area of southwestern china. Environmental Earth Sciences, 74(8), 6407-6420.

14. Wang, S.; Zhang, L.; Zhang, H.; Han, X.; Zhang, L. Spatial-Temporal Wetland Landcover Changes of Poyang Lake Derived from Landsat and HJ-1A/B Data in the Dry Season from 1973-2019. Remote Sens. 2020, 12, 1595.

15. Szabó, L.; Deák, B.; Bíró, T.; Dyke, G.J.; Szabó, S. NDVI as a Proxy for Estimating Sedimentation and Vegetation Spread in Artificial Lakes-Monitoring of Spatial and Temporal Changes by Using Satellite Images Overarching Three Decades. Remote Sens. 2020, 12, 1468.

16. Malekmohammadi, B. , \& Blouchi, L. R. . (2014). Ecological risk assessment of wetland ecosystems using multi criteria decision making and geographic information system. Ecological Indicators, 41(6), 133-144.

17. Lane, C. , Liu, H. , Autrey, B. , Anenkhonov, O. , Chepinoga, V. , \& Wu, Q. . (2014). Improved wetland classification using eight-band high resolution satellite imagery and a hybrid approach. Remote Sensing, 6(12), 12187-12216.

18. Julie Betbeder, Sébastien Rapinel, Samuel Corgne, Eric Pottier, Laurence Hubert-Moy,et al. (2015). Terrasar$x$ dual-pol time-series for mapping of wetland vegetation. ISPRS Journal of Photogrammetry and Remote Sensing, 107, S0924271615001380.

19. Tian, Y.; Jia, M.; Wang, Z.; Mao, D.; Du, B.; Wang, C. Monitoring Invasion Process of Spartina alterniflora by Seasonal Sentinel-2 Imagery and an Object-Based Random Forest Classification. Remote Sens. 2020, 12, 1383.

20. Franklin, S. E. , Skeries, E. M. , Stefanuk, M. A. , \& Ahmed, O. S. . (2018). Wetland classification using radarsat-2 SAR quad-polarization and landsat-8 OLI spectral response data: a case study in the hudson bay lowlands ecoregion. International Journal of Remote Sensing, 39(6), 1615-1627. 
21. Villoslada M, Bergamo T F, Ward R D, et al.(2020). Fine scale plant community assessment in coastal meadows using UAV based multispectral data. Ecological Indicators, , 111: 105979.

22. Cao, J.; Leng, W.; Liu, K.; Liu, L.; He, Z.; Zhu, Y. Object-Based Mangrove Species Classification Using Unmanned Aerial Vehicle Hyperspectral Images and Digital Surface Models. Remote Sens. 2018, 10, 89.

23. Tao Liu, Amr Abd-Elrahman. (2018). Multi-view object-based classification of wetland land covers using unmanned aircraft system images. Remote Sensing of Environment, Volume 216; Pages 122-138.

24. Munyati, C. , Ratshibvumo, T. , \& Ogola, J. . (2013). Landsat tm image segmentation for delineating geological zone correlated vegetation stratification in the kruger national park, south africa. Physics and Chemistry of the Earth, Parts A/B/C, 55-57(Complete), 1-10.

25. Churches, C. E. , Wampler, P. J. , Sun, W. , \& Smith, A. J. . (2014). Evaluation of forest cover estimates for haiti using supervised classification of landsat data. International Journal of Applied Earth Observation and Geoinformation, 30, 203-216.

26. Gerke, M. , \& Xiao, J. . (2014). Fusion of airborne laserscanning point clouds and images for supervised and unsupervised scene classification. ISPRS Journal of Photogrammetry and Remote Sensing, 87, 78-92.

27. Maulik, U., \& Chakraborty, D. (2013). Learning with transductive SVM for semisupervised pixel classification of remote sensing imagery. ISPRS journal of photogrammetry and remote sensing, 77, 66-78.

28. Crasto, N. , Hopkinson, C. , Forbes, D. L. , Lesack, L. , Marsh, P. , \& Spooner, I. , et al. (2015). A lidar-based decision-tree classification of open water surfaces in an arctic delta. Remote Sensing of Environment, 164, 90-102.

29. O'Neil, G. L. , Goodall, J. L. , \& Watson, L. T. . (2018). Evaluating the potential for site-specific modification of lidar dem derivatives to improve environmental planning-scale wetland identification using RF classification. Journal of Hydrology, 559.

30. Howard, A. G. . (2013). Some improvements on deep CNN based image classification. Computer Science.

31. Yao, X., Yang, H., Wu, Y., Wu, P., Wang, B., Zhou, X., \& Wang, S. (2019). Land use classification of the deep convolutional neural network method reducing the loss of spatial features. Sensors,19(12), 2792.

32. Yang, C. , Rongshuang, F. , Xiucheng, Y. , Jingxue, W. , \& Aamir, L. (2018). Extraction of urban water bodies from high-resolution remote-sensing imagery using deep learning. Water, 10(5), 585.

33. Yuan, \& Jiangye. (2017). Learning building extraction in aerial scenes with convolutional networks. IEEE Transactions on Pattern Analysis and Machine Intelligence, 1-1.

34. Gu, J. , Wang, Z. , Kuen, J. , Ma, L. , Shahroudy, A. , \& Shuai, B. , et al. (2015). Recent advances in CNN. Computer Science.

35. Srinivas, S. , Subramanya, A. , \& Babu, R. V. . (2016). Training sparse neural networks.

36. Liang, S. , Lan, Y. , Jiang, S. , Li, Y. , \& Lu, Z. . (2017). The activities of microbial communities in huixian wetland sediments under the interactive toxicity of $\mathrm{cu}(\mathrm{ii})$ and pentachloronitrobenzene. Acta Ecologica Sinica, 37(6), 379-391.

37. Feng, W. . (2017). Fish diversity in huixian wetland in guangxi. Wetland Science, 15(2), 256-262.

38. Breiman L. RFs[J]. Machine Learning ,2001,45 (1):5-32.

39. Mutanga, O., Adam, E., \& Cho, M. A. (2012). High density biomass estimation for wetland vegetation using WorldView-2 imagery and random forest regression algorithm. International Journal of Applied Earth Observation and Geoinformation,18, 399-406.

40. Van Beijma, S., Comber, A., \& Lamb, A. (2014). Random forest classification of salt marsh vegetation habitats using quad-polarimetric airborne SAR, elevation and optical RS data. Remote Sensing of Environment,149, 118-129.

41. Badrinarayanan, V., Handa, A., \& Cipolla, R. (2015). Segnet: A deep convolutional encoder-decoder architecture for robust semantic pixel-wise labelling. arXiv preprint arXiv:1505.07293.

42. Ioffe, S., \& Szegedy, C. (2015). Batch normalization: Accelerating deep network training by reducing internal covariate shift. In International conference on machine learning(pp. 448-456). PMLR.

43. Long, J., Shelhamer, E., \& Darrell, T. (2015). Fully convolutional networks for semantic segmentation. In Proceedings of the IEEE conference on computer vision and pattern recognition (pp. 3431-3440)..

44. Chen, L. C., Barron, J. T., Papandreou, G., Murphy, K., \& Yuille, A. L. (2016). Semantic image segmentation with task-specific edge detection using cnns and a discriminatively trained domain transform. In Proceedings of the IEEE conference on computer vision and pattern recognition (pp. 4545-4554).

45. Eigen, D., \& Fergus, R. (2015). Predicting depth, surface normals and semantic labels with a common multiscale convolutional architecture. In Proceedings of the IEEE international conference on computer vision (pp. 2650-2658). 
46. Hu, K., Zhang, S., \& Zhao, X. (2019). Context-based conditional random fields as recurrent neural networks for image labeling. Multimedia Tools and Applications, 1-11.

47. Dronova, I. (2015). Object-based image analysis in wetland research: A review. Remote Sensing, 7(5), 63806413.

48. Liu, F., \& Fang, M. (2020). Semantic Segmentation of Underwater Images Based on Improved Deeplab. Journal of Marine Science and Engineering,8(3), 188.

49. Zhang, Z., \& Sabuncu, M. R. (2018). Generalized cross entropy loss for training deep neural networks with noisy labels. arXiv preprint arXiv:1805.07836.

50. Ruder, S. (2016). An overview of gradient descent optimization algorithms. arXiv preprint arXiv:1609.04747.

51. Song, S., Liu, J., Liu, Y., Feng, G., Han, H., Yao, Y., \& Du, M. (2020). Intelligent Object Recognition of Urban Water Bodies Based on Deep Learning for Multi-Source and Multi-Temporal High Spatial Resolution Remote Sensing Imagery. Sensors, 20(2), 397.

52. Sun, G., Huang, H., Zhang, A., Li, F., Zhao, H., \& Fu, H. (2019). Fusion of multiscale convolutional neural networks for building extraction in very high-resolution images. Remote Sensing, 11(3), 227.

53. Pouliot, D., Latifovic, R., Pasher, J., \& Duffe, J. (2019). Assessment of convolution neural networks for wetland mapping with landsat in the central Canadian boreal forest region. Remote Sensing,11(7), 772.

54. Rezaee, M., Mahdianpari, M., Zhang, Y., \& Salehi, B. (2018). Deep convolutional neural network for complex wetland classification using optical remote sensing imagery. IEEE Journal of Selected Topics in Applied Earth Observations and Remote Sensing,11(9), 3030-3039.

55. Fu, B., Wang, Y., Campbell, A., Li, Y., Zhang, B., Yin, S., ... \& Jin, X. (2017). Comparison of object-based and pixel-based Random Forest algorithm for wetland vegetation mapping using high spatial resolution GF-1 and SAR data.Ecological indicators,73, 105-117.

56. Zhao, H. H., \& Liu, H. (2020). Multiple classifiers fusion and CNN feature extraction for handwritten digits recognition. Granular Computing, 5(3), 411-418.

57. Hu, Y., Zhang, J., Ma, Y., Li, X., Sun, Q., \& An, J. (2019). Deep learning classification of coastal wetland hyperspectral image combined spectra and texture features: A case study of Huanghe (Yellow) River Estuary wetland. Acta Oceanologica Sinica,38(5), 142-150.

58. Wang, M., Fei, X., Zhang, Y., Chen, Z., Wang, X., Tsou, J. Y., ... \& Lu, X. (2018). Assessing texture features to classify coastal wetland vegetation from high spatial resolution imagery using completed local binary patterns (CLBP).Remote Sensing,10(5), 778.

59. Szantoi, Z. , Escobedo, F. , Abd-Elrahman, A. , Smith, S. , \& Pearlstine, L. . (2013). Analyzing fine-scale wetland composition using high resolution imagery and texture features. International Journal of Applied Earth Observation and Geoinformation, 23(8), 204-212. 